BMJ

Open

Gastroenterology

\title{
Impact of feedback and monitoring on colonoscopy withdrawal times and polyp detection rates
}

\author{
Amalie Bach Nielsen, Ole Haagen Nielsen, Jakob Hendel
}

To cite: Nielsen $A B$, Nielsen $\mathrm{OH}$, Hendel J. Impact of feedback and monitoring on colonoscopy withdrawal times and polyp detection rates. BMJ Open Gastro 2017:4:e000142.

doi:10.1136/bmjgast-2017000142

Additional material is published online only. To view please visit the journal online (http://dx.doi.org/10. 1136/bmjgast-2017-000142)

Received 2 March 2017 Revised 27 April 2017 Accepted 16 May 2017
Department of

Gastroenterology, Herlev Hospital, University of Copenhagen, Denmark

\section{Correspondence to} Professor Ole Haagen Nielsen, Department of Gastroenterology D112, Herlev Hospital, University of Copenhagen, Herlev Ringvej 75, Herlev DK-2730, Denmark; ole.haagen. nielsen@regionh.dk

\section{ABSTRACT}

Background: Previous studies have shown colonoscopy withdrawal time (WT) to be a reliable surrogate indicator for polyp detection rate (PDR) and adenoma detection rate (ADR) in colonoscopy. Our aim was to assess the impact of feedback and monitoring of WT on PDR in routine colonoscopies with long-term follow-up.

Materials and methods: A total of 307

colonoscopies were performed in three separate clinical scenarios. First, PDR and WT were recorded without the staff being aware of the specific objective of the study. Before the second scenario, the staff was given interventional information and feedback on WTs and PDRs from the first scenario and was encouraged to aim for a minimum WT of 8 min. Retention of knowledge gained was reassessed in the third scenario 1 year later. Results: The PDR in the first two scenarios differed significantly $(p<0.01)$, with a more than $90 \%$ increase in PDR after intervention from $22 \%$ to $42 \%(95 \% \mathrm{Cl}$ 1.44 to 4.95$)$, although the mean WT did not change (6.8 vs $7.2 \mathrm{~min} ; p>0.05$ ). The increase in PDR between the first and second scenarios was retained in the third follow-up scenario 1 year later where the WT of both polyp-positive and polyp-negative colonoscopies was found to be longer.

Conclusions: PDR almost doubled from the first to the second scenario of a real-life colonoscopy setting, indicating that awareness of WT is crucial. The knowledge gained from this intervention in routine practice was even retained after a year.

\section{INTRODUCTION}

Worldwide, colorectal cancer (CRC) is the third most common cancer in men and second in women, and it is responsible for an estimated $8.5 \%$ of deaths from cancer. ${ }^{1}$ Thus $\sim 1.3$ million people are diagnosed yearly with CRC, and almost 700000 deaths occur from CRC every year. ${ }^{2}{ }^{3}$ However, efforts towards improving quality and accuracy of colonoscopies are of vital importance, ${ }^{4}$ as polypectomy is a procedure known to decrease the incidence of CRC by 76-90\%. ${ }^{5}$

The Danish Colorectal Cancer Screening Programme was implemented in 2014 and is

\section{Summary box}

What is already known about this subject?

- Worldwide, colorectal cancer (CRC) is the second most common cancer in women and third in men.

- Thus, the significance of colonic polyp-to-cancer pathway makes the reliability of colonoscopy examinations of paramount importance.

- Efforts towards improving quality and accuracy of colonoscopies are of vital importance, as polypectomy may decrease the incidence of CRC by more than $75 \%$.

What are the new findings?

- Monitoring and feedback on colonoscopy withdrawal times significantly increase polyp detection rate (PDR) in a clinical setting with no correlation with withdrawal time (WT).

- The choice of feedback parameter was found to be of less importance.

- A "corporate memory" of the significance of the quality of the colonoscopy was imprinted with a short, focused feedback session and remained stable after a year.

How might it impact on clinical practice in the foreseeable future?

- A quality-improving feedback strategy used in routine practice of screening and surveillance colonoscopies may lead to improved outcome of examinations in other real-life colonoscopy settings.

offered every second year to all citizens aged 50-74 years using an immunochemical test of faeces for blood. Despite the fact that colonoscopy is economically and specificity efficient, it is crucial to minimise false-negative results. Previous studies have revealed that up to $5 \%$ of all CRCs are missed at colonoscopy; $^{7}$ this also applies for up to $26 \%$ of adenomas as well as $27 \%$ of polyps. ${ }^{489}$ Since it might take up to 20 years to develop CRC from certain mutations of tumour-suppressor genes and oncogenes, ${ }^{10}$ an optimally performed colonoscopy-and thus detection rate-is central to efforts to prevent CRC. 
The polyp detection rate (PDR), which indicates the percentage of colonoscopies with one or more polyps identified, and the adenoma detection rate (ADR), which is the percentage of colonoscopies with one or more adenomas identified, are two crucial parameters in CRC screening. A high ADR is associated with a decreased mortality and postcolonoscopy colorectal cancer (PCCRC) incidence. ${ }^{11}$ Thresholds of minimum $20 \%$ and minimum $25 \%$ of ADR and PDR, respectively, have earlier been advocated. ${ }^{7}$

Previous studies on PDR/ADR have focused on the endoscopists by tracking individual PDRs and ADRs. However, our endoscopy centre at a tertiary referral hospital has a long-standing tradition of considering endoscopy to be a team effort that emphasises the role of the nursing staff in polyp detection. Given the centralisation of CRC treatment and implementation of the Danish Colorectal Cancer Screening Programme, the case mix in our unit was expected to shift significantly towards a higher incidence of colonic polyps.

Colonoscopy withdrawal time (WT) is considered a well-established and reliable surrogate quality indicator for ADR/PDR, and focus on WT should accordingly be implemented as a standard in routine colonoscopy. ${ }^{12-15}$ WT is defined as the time spent investigating the intestinal mucosa for pathological changes during withdrawal of the colonoscope from the caecum towards the anus. As most polyps are detected during the withdrawal phase, this part of the colonoscopy procedure is considered of higher importance than the insertion phase. ${ }^{6}$ Nevertheless, some studies have highlighted other factors for improvement of colonoscopy detection as well, for example, the importance of bowel preparation, caecal intubation, experience of individual endoscopists, withdrawal technique, sedation and time of day for the examination. ${ }^{16}{ }^{17}$ In one of the studies, associations between proximal serrated polyp detection and a number of quality factors were tested. ${ }^{17}$ In this study, WT was the only significant factor, whereas patient age, sex, intubation time, withdrawal techniques and time of day were not. The US Multi-Society Task Force on Colorectal Cancer advises a minimum of $6 \mathrm{~min}$ for optimal mean WT in routine colonoscopy, ${ }^{18}$ whereas more recent studies have recommended a WT in the range of 6-9 min. ${ }^{12} 1920$

The aim of this study was to evaluate whether monitoring information and feedback on colonoscopy WT to the endoscopy team of colonoscopists and nursing staff may have an impact on PDR in a clinical setting at a large endoscopy unit of a tertiary referral centre.

\section{METHODS}

\section{Colonoscopy material}

A total of 314 patients (53\% male patients, $47 \%$ female patients) with an adequate colon preparation undergoing routine colonoscopy at the Endoscopy Centre, Herlev Hospital, University of Copenhagen, were enrolled in this prospective study. Most examinations performed were screening or surveillance colonoscopies. Seven patients were excluded. Exclusion criteria included apart from inadequate cleansing, incomplete examinations and inconsistent data on the survey form completed by the assisting nurse during each individual colonoscopy procedure. Thus, 307 patients were eligible to be included into the study.

Each patient was prepared for examination using standard bowel preparation (MoviPrep, Norgine BV, Amsterdam, The Netherlands) and was provided oral and written information for all components of the colonoscopy preparation, and the importance of compliance with bowel cleansing was emphasised. The endoscopy procedures were performed using Evis Exera III colonoscopes (Olympus Europe, Hamburg, Germany).

All endoscopists had performed a minimum of 500 colonoscopy examinations prior to this study.

\section{Monitoring and feedback}

Three phases of the study were carried out in an assessment to increase the PDR of colonoscopies. The first study scenario was performed from August to September 2015, the second from October to November 2015, and the third from October to November 2016. During these three phases, time spent on the different phases of the procedures was recorded by the trained assistant nurse who additionally carefully took part in each colonoscopy by watching the video screen together with the endoscopist during the withdrawal phase. This included a survey form with time points for procedure start, time for reaching the caecum, initiation of withdrawal and completion of the procedure. Furthermore, time spent on removal of polyps was registered. Time points were noted as the actual clock time. Neither number, types, nor sizes of polyps were recorded, so as to avoid disclosure of the main objective and to introduce any bias into the first phase. All colonoscopy procedures were performed as standard practice with no changes during the three study phases. WT was calculated for each procedure as time from caecum to anus in minutes-adjusted for any polyp removal, if applicable.

Before the first phase, the endoscopists and nurses were informed that the survey was part of a general benchmarking of time usage in colonoscopy procedures at the endoscopy centre. At this point, the staff received no information about our interest in PDR or WT, and the survey form was designed in such a manner that the purpose of the information collected was not transparent. However, before the second phase, an intervention took place in the form of separate team meetings of the nurses and the endoscopists at which the aim of the study was clearly disclosed. The entire staff was informed about the monitoring of WT and PDR, and anonymous feedback on these parameters was provided. Further, the staff was informed that WT and PDR results would be compared with the general recommendations of a $6 \mathrm{~min}$ 
$\mathrm{WT}^{21}$ and a $40 \%$ PDR. $^{22}$ Based on results from the first study phase, the endoscopic teams were encouraged to aim for a minimum WT of $8 \mathrm{~min}$.

After the second phase, the entire staff was informed of the results, and the importance of PDR was stressed.

One year after the second phase, a third phase analogue to the second scenario but without any introduction was performed to test long-term awareness with respect to the importance of PDR and WT. During this year, there had been minor turnover between different teams, but no changes in endoscopists nor the endoscopic staff as such.

All polyps were retrieved for histology to assess the ADR.

\section{Statistical analysis}

Overall, examination time, insertion time, and WT were calculated based on the time points noted in the survey forms. A significant difference between $W T \geq 8$ min and WT $<8$ min was found using a binary $2 \times 2$ table, a twosided $z$-test, and a $\chi^{2}$ test. PDRs of the second and third study phases were compared to the PDR of the first phase using a binary $2 \times 2$ table, a two-sided $z$-test, and a $\chi^{2}$ test. By assuming normality based on the distribution of the WTs, significant differences between the mean WT of the study groups were evaluated by a two-tailed $t$-test. A p value of 0.05 was considered statistically significant.

Using a power calculation with independent study groups and the PDR of the first phase without knowledge (conventional colonoscopy) assumed to be $40 \%$ based on prior experience from small observational series within our group of patients and of the second phase set to $60 \%$ which is actually the benchmark of the Danish Colorectal Cancer Screening Programme, and with a type I error of 0.05 and a power of $80 \%$, the sample size was a minimum of 97 patients in each arm. We chose, however, to include no fewer than 100 patients in each phase, with the third phase basically being a replication of the second.

\section{Ethics approval}

The protocol was assessed by the local ethics board and a formal approval was not found to be required as the aim of the project was quality assessment at an endoscopic university centre with no individual patient data recorded.

\section{RESULTS}

From the analysis of the total of 307 colonoscopies, the WTs varied from 2 to $26 \mathrm{~min}$. One or more polyps were found in 22 of the 100 colonoscopies in the first study phase, 43 of 102 colonoscopies in the second phase and 53 of 105 colonoscopies in the 1-year follow-up. The PDR differed significantly $(p<0.01)$ between the first and second study phases, with a more than $90 \%$ increase in PDR after intervention from 22\% to $42 \%$ (95\% CI 1.44 to 4.95$)$. ADR increased similarly from $14 \%$ to $33 \%$ $(\mathrm{p}<0.01 ; 95 \%$ CI 1.50 to 6.11$)$. The prevalence of advanced neoplasia was $4 \%$ and remained unchanged during the study. A trend towards increased mean PDR for most WTs in the second study phase was revealed, as illustrated in figure 1 . However, the mean WT did not change (6.8 vs $7.2 \mathrm{~min}$; $\mathrm{p}>0.05$ ) despite the instructions given to all endoscopists involved before initiating the second study phase. Compliance with the 6 min WT recommendation increased significantly from $53 \%$ in the first study phase to $71 \%$ in the second study phase ( $\mathrm{p}<0.01 ; 95 \%$ CI 1.21 to 3.89$)$, while compliance with the 8 min WT assessment increased from $34 \%$ to $44 \%$, which was not statistically significant $(\mathrm{p}=0.15 ; 95 \%$ CI 0.86 to 2.70$)$.

No significant changes in PDR or ADR were, however, observed between the second and the third 1-year follow-up phase, although the mean WT of polyp-positive and polyp-negative colonoscopies was found significantly higher in the 1-year follow-up (7.9 vs $11.9 \mathrm{~min}(\mathrm{p}<0.05)$; 6.6 vs $9.9 \mathrm{~min}(\mathrm{p}<0.05))$. Distribution of bowel preparation within the groups of patients graded on a 4-point scale (ie, optimal, good, acceptable, or unacceptable,
Figure 1 Mean polyp detection rate (PDR) at each specific withdrawal time (minutes) of the three study phases.

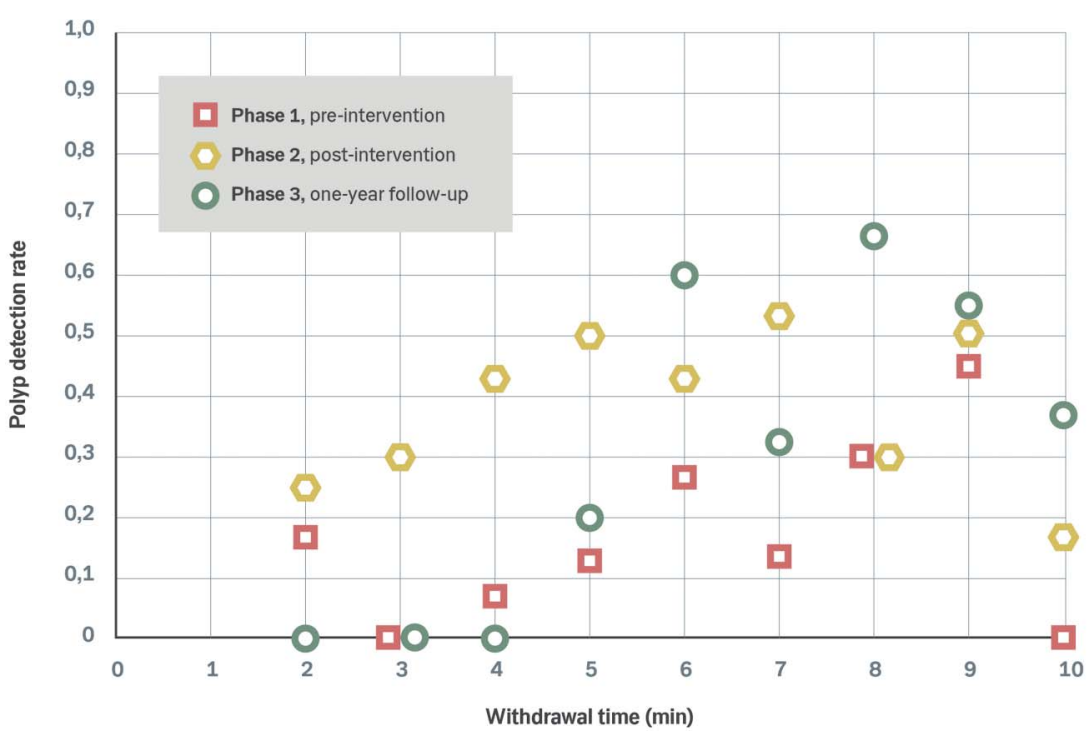


Table 1 Mean polyp detection rate, adenoma detection rate, colonoscopy withdrawal time and gender distribution of the three study phases

\begin{tabular}{|c|c|c|c|c|c|c|c|c|c|}
\hline & $\begin{array}{l}\text { Phase } 1 \\
(n=100)(\%)\end{array}$ & $\begin{array}{l}\text { Phase } 2 \\
(n=102)(\%)\end{array}$ & OR & $95 \% \mathrm{Cl}$ & p Value & $\begin{array}{l}\text { Phase } 3 \\
(n=105)(\%)\end{array}$ & OR & $95 \% \mathrm{Cl}$ & p Value \\
\hline Female sex (\%) & 60 & 43 & 0.50 & 0.29 to 0.88 & $<0.02$ & 38 & 0.41 & 0.23 to 0.72 & $<0.01$ \\
\hline PDR (\%) & 22 & 43 & 2.67 & 1.44 to 4.96 & $<0.01$ & 50 & 3.55 & 1.92 to 6.55 & $<0.01$ \\
\hline ADR (\%) & 14 & 33 & 3.03 & 1.50 to 6.11 & $<0.01$ & 37 & 3.61 & 1.80 to 7.23 & $<0.01$ \\
\hline Mean WT (min) & 6.8 & 7.2 & & & 0.73 & 10.9 & & & $<0.01$ \\
\hline $\mathrm{WT} \geq 6 \mathrm{~min}$ & 53 & 71 & 2.17 & 1.21 to 3.89 & $<0.01$ & 87 & 5.93 & $2.94-11.98$ & $<0.01$ \\
\hline $\mathrm{WT} \geq 8 \mathrm{~min}$ & 34 & 44 & 1.53 & 0.86 to 2.70 & 0.15 & 74 & 5.52 & 3.00 to 10.16 & $<0.01$ \\
\hline
\end{tabular}

where the last grading always leads to a renewed examination) was similar when comparing the three phases of the study (data not shown).

All data for WTs, PDRs, ADRs, and gender distribution are summarised in table 1.

\section{DISCUSSION}

We examined whether PDR and subsequently ADR could be increased using the WT as a surrogate marker via the Hawthorne effect (knowing that the WT was assessed) and feedback (provided after the first study phase). Furthermore, the retention of knowledge gained within the organisation was assessed by a 1-year follow-up.

Information and feedback after the first phase had a significant impact on PDR, which almost doubled (22\% vs $42 \%$ ). The endoscopists were focusing on PDR through the surrogate marker (WT) and the feedback given. The PDRs increased at all levels of monitored WTs. The mean WT did, however, not increase significantly in between the first and second phases. Thus, monitoring and feedback appears to be crucial factors for the increase in PDR and not the measured parameter (ie, the WT itself). The increase in PDR was additionally mirrored in an increased ADR. The increased PDR/ADR was found to be maintained, and thus the endoscopy team was able to maintain focus on the importance of PDR more than a year after the original intervention. Further, there was a tendency towards an increase in WT without an effect on ADR which is in agreement with previous data showing a limit to increase ADR by increasing WT, as the incremental increase becomes useless and the time spent prohibitive. ${ }^{14}$ In this respect, our results signify that monitoring of WT exerts its effect mainly by a Hawthorne effect because we did not observe any significant increase in WT from the first study phase to the second.

The strength of this study lies in its focus on the performance of the endoscopy team as a whole in a real-life colonoscopy setting. In our standard setup for colonoscopy which was unchanged between phase 1 and phase 3 , we have two video screens-one for the endoscopist and one for the assistant nurse. The assistant nurse is required to aid the endoscopist to spot any suspected pathology, including polyps, during retraction of the endoscope. Further, the blinded initial assessment of PDR in the first phase gives an accurate image of the team's performance without any Hawthorne effect. However, the study also has some limitations because no descriptive data on the procedures were recorded on the survey form. This could influence the purpose by revealing the focus of the first part of the study prior to the subsequent intervention phase. This circumstance also precludes evaluation of other potential factors that could influence the results, for example, interobserver variation in PDR, colonoscopy experience and specific withdrawal techniques. Also the use of ADR for polyp detection would have been a correct measure, but would also have emphasised the importance of ADR as the final goal prior to intervention. For similar reasons no analysis of type, size, location or number of polyps and adenomas detected was undertaken. Thus, we performed retrospective analysis of the impact of these factors, but the number of patients included into this study did not allow for making any further definite conclusions.

Despite some restrictions, including number of colonoscopies done over the three separate phases, as well as the low compliance with the $8 \mathrm{~min}$ WT, some interesting tendencies were, however, revealed. The optimal PDR appears to be achieved shortly after the 6 min recommended WT. Even at 2 and 3 min WT cut points, there was a trend towards higher mean PDRs than the mean PDR of the first study phase (figure 1). These results imply that providing feedback in itself might be sufficient to increase the PDRs of the endoscopists, as reflected in the second study phase.

Two studies have found a similar effect of monitoring in itself, but both studies focused on individual endoscopists rather than the entire endoscopy team. ${ }^{21}{ }^{23}$ In contrast, several studies have revealed that an increased WT is essential and raises PDR/ADR, especially for small, flat

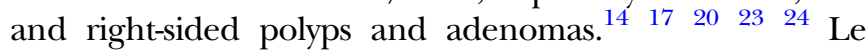
Clercq et $a t^{25}$ showed that such lesions are the most frequently missed in everyday practice, which subsequently might lead to PCCRC. Moreover, it was observed that $86 \%$ of all PCCRCs were preventable due to procedural factors.

One study reported an ADR increase from $36 \%$ to $47 \%(\mathrm{p}=0.001)$ after a 2-hour training in withdrawal 
techniques and colonoscopy quality indicators together with a monthly ADR feedback, whereas ADR remained unchanged for the untrained group of endoscopists. ${ }^{4}$ In a study of 541 screening colonoscopies among 10 endoscopists, who were informed about guidelines and published data on WT and PDR, these factors were monitored. ${ }^{26}$ Each endoscopist was subsequently given feedback once every 3-6 months, and a trend towards an increased PDR and neoplasia detection was revealed, supporting the positive influence of feedback on PDR.

In a very recent trial, researchers took a new approach to test whether WT is positively associated with detection. ${ }^{15}$ In this prospective trial with four highly skilled endoscopists, who each performed 50 colonoscopies, 200 patients were randomised to undergo colonoscopy with a WT of 3 or $6 \mathrm{~min}$, followed by a second withdrawal lasting $6 \mathrm{~min}$ in both study arms. The calculated miss rate for adenomas was higher in the $3 \mathrm{~min}$ withdrawal group compared with the 6 min group ( 48 vs $23 \%$ ). The ADR was similar between the groups (39 vs $41 \%$ ), but the number of adenomas detected per colonoscopy was lower in the $3 \mathrm{~min}$ group as compared with the $6 \mathrm{~min}$ group $(0.55$ vs 0.80$)$. Overall this study provides strong evidence for establishing minimum average WTs.

However, higher PDRs/ADRs lead to more surveillance colonoscopies and thus higher colonoscopy-related costs. A recent study has indicated, through a microsimulation model, that higher ADRs in screening colonoscopies (mean ADR of $15 \%$ vs mean ADR of $39 \%$ ) reduce lifetime CRC incidence and mortality by $53 \%$ to $60 \% .^{27}$ Remarkably, this outcome was obtained without higher overall expenses (US\$2.1 million vs $\$ 1.8$ million) due to averted cancer treatment costs.

\section{CONCLUSIONS}

The present study shows that monitoring and feedback on colonoscopy withdrawal times significantly increase PDRs in a clinical setting with no correlation with WTs. PDR almost doubled from the first to the second study phase, which leads to the conclusion that simply monitoring colonoscopy and giving feedback to the endoscopy team is crucial to polyp detection. However, the choice of feedback parameter seems to be of less importance. Furthermore, it was possible for usgranted with a dedicated staff-to imprint 'corporate memory' of the significance of the quality of the colonoscopy with a short, focused feedback session. The effect of this corporate memory was shown to remain stable after a year. Thus, a quality-improving feedback strategy used in routine practice of screening and surveillance colonoscopies may lead to improved examinations in other real-life colonoscopy settings as well.

Acknowledgements We thank Ms Lisa Rohbach for skilful secretarial assistance.

Contributors $\mathrm{JH}$ and $\mathrm{OHN}$ were involved in protocol design. ABN took part in execution of the study and prepared the first manuscript version after analysis and presentation of study results performed in collaboration with OHN and $\mathrm{JH}$. All authors approved the final version of the manuscript.
Competing interests None declared.

Ethics approval Local ethics board at Herlev Hospital.

Provenance and peer review Not commissioned; externally peer reviewed.

Data sharing statement Original data from the survey forms of each of the three study scenarios are available from the corresponding author.

Open Access This is an Open Access article distributed in accordance with the Creative Commons Attribution Non Commercial (CC BY-NC 4.0) license, which permits others to distribute, remix, adapt, build upon this work noncommercially, and license their derivative works on different terms, provided the original work is properly cited and the use is non-commercial. See: http:// creativecommons.org/licenses/by-nc/4.0/

\section{REFERENCES}

1. Ferlay J, Soerjomataram I, Dikshit R, et al. Cancer incidence and mortality worldwide: Sources, methods and major patterns in GLOBOCAN 2012. Int J Cancer 2015;136:E359-86.

2. Brenner H, Kloor M, Pox CP. Colorectal cancer. Lancet 2014;383:1490-502.

3. Torre LA, Bray F, Siegel RL, et al. Global cancer statistics, 2012. CA Cancer J Clin 2015;65:87-108.

4. Coe SG, Crook JE, Diehl NN, et al. An endoscopic quality improvement program improves detection of colorectal adenomas. Am J Gastroenterol 2013;108:219-26.

5. Brenner H, Chang-Claude J, Seiler CM, et al. Protection from colorectal cancer after colonoscopy: a population-based, case-control study. Ann Intern Med 2011;154:22-30.

6. Barclay RL, Vicari JJ, Doughty AS, et al. Colonoscopic withdrawal times and adenoma detection during screening colonoscopy. $N$ Engl J Med 2006;355:2533-41.

7. Moritz V, Bretthauer M, Ruud HK, et al. Withdrawal time as a quality indicator for colonoscopy - a nationwide analysis. Endoscopy 2012;44:476-81.

8. Morini S, Hassan C, Zullo A, et al. Detection of colonic polyps according to insertion/withdrawal phases of colonoscopy. Int $\mathrm{J}$ Color Dis 2009;24:527-30.

9. Sawhney MS, Cury MS, Neeman N, et al. Effect of institution-wide policy of colonoscopy withdrawal time $>$ or $=7$ minutes on polyp detection. Gastroenterology 2008;135:1892-8.

10. Rex DK. Optimal withdrawal and examination in colonoscopy. Gastroenterol Clin North Am 2013:429-42.

11. Kaminski MF, Regula J, Kraszewska E, et al. Quality indicators for colonoscopy and the risk of interval cancer. $N$ Engl J Med 2010;362:1795-803.

12. Barclay RL, Vicari JJ, Greenlaw RL. Effect of a time-dependent colonoscopic withdrawal protocol on adenoma detection during screening colonoscopy. Clin Gastroenterol Hepatol 2008;6:1091-8.

13. Rex DK, Bond JH, Winawer S, et al. Quality in the technical performance of colonoscopy and the continuous quality improvement process for colonoscopy: recommendations of the U.S. Multi-Society Task Force on Colorectal Cancer. Am J Gastroenterol 2002;97:1296-308.

14. Lee TJ, Blanks RG, Rees CJ, et al. Longer mean colonoscopy withdrawal time is associated with increased adenoma detection: evidence from the Bowel Cancer Screening Programme in England. Endoscopy 2013;45:20-6.

15. Kumar S, Thosani N, Ladabaum U, et al. Adenoma miss rates associated with a 3-minute versus 6-minute colonoscopy withdrawal time: a prospective, randomized trial. Gastrointest Endosc 2017:85:1273-80.

16. Lee TJ, Rees CJ, Blanks RG, et al. Colonoscopic factors associated with adenoma detection in a national colorectal cancer screening program. Endoscopy 2014;46:203-11.

17. de Wijkerslooth TR, Stoop EM, Bossuyt PM, et al. Differences in proximal serrated polyp detection among endoscopists are associated with variability in withdrawal time. Gastrointest Endosc 2013;77:617-23.

18. Rex DK. Colonoscopy withdrawal times and adenoma detection rates. Gastroenterol Hepatol (N Y) 2007;3:609-10.

19. Overholt BF, Brooks-Belli L, Grace M, et al. Withdrawal times and associated factors in colonoscopy: a quality assurance multicenter assessment. J Clin Gastroenterol 2010;44:80-6.

20. Butterly L, Robinson CM, Anderson JC, et al. Serrated and adenomatous polyp detection increases with longer withdrawal time: results from the New Hampshire Colonoscopy Registry. Am J Gastroenterol 2014;109:417-26. 
21. Rex DK. Colonoscopic withdrawal technique is associated with adenoma miss rates. Gastrointest Endosc 2000;51:33-6.

22. Calderwood AH, Jacobson BC. Colonoscopy quality: metrics and implementation. Castroenterol Clin North Am 2013;42:599-618.

23. Simmons DT, Harewood GC, Baron TH, et al. Impact of endoscopist withdrawal speed on polyp yield: implications for optimal colonoscopy withdrawal time. Aliment Pharmacol Ther 2006:24:965-71.

24. Pickhardt PJ, Nugent PA, Mysliwiec PA, et al. Location of adenomas missed by optical colonoscopy. Ann Intern Med 2004;141:352-9.
25. le Clercq CM, Bouwens MW, Rondagh EJ, et al. Postcolonoscopy colorectal cancers are preventable: a population-based study. Gut 2014;63:957-63.

26. Lin OS, Kozarek RA, Arai A, et al. The effect of periodic monitoring and feedback on screening colonoscopy withdrawal times, polyp detection rates, and patient satisfaction scores. Gastrointest Endosc 2010;71:1253-9.

27. Meester RG, Doubeni CA, Lansdorp-Vogelaar I, et al. Variation in adenoma detection rate and the lifetime benefits and cost of colorectal cancer screening: a microsimulation model. JAMA 2015;313:2349-58. 